\title{
Targeted gadolinium-loaded dendrimer nanoparticles for tumor-specific magnetic resonance contrast enhancement
}

\author{
Scott D Swanson' \\ Jolanta F Kukowska-Latallo \\ Anil K Patri ${ }^{5}$ \\ Chunyan Chen ${ }^{6}$ \\ Song $\mathrm{Ge}^{4}$ \\ Zhengyi $\mathrm{CaO}^{3}$ \\ Alina Kotlyar ${ }^{3}$ \\ Andrea T East ${ }^{7}$ \\ James R Baker ${ }^{3}$ \\ 'Department of Radiology, The \\ University of Michigan Medical School, \\ ${ }^{2}$ Department of Internal Medicine, \\ The University of Michigan Medical \\ School, ${ }^{3}$ Michigan Nanotechnology \\ Institute for Medicine and Biological \\ Sciences, The University of Michigan, \\ ${ }^{4}$ Applied Physics, The University of \\ Michigan, MD, USA; ${ }^{5}$ Present address: \\ National Cancer Institute at Frederick \\ (Contractor), MD, USA; ${ }^{6}$ Present \\ address: Intel Corporation, Chandler, \\ AZ, USA; ${ }^{\text {PPresent }}$ address: Stritch \\ School of Medicine, Chicago, ILL, USA
}

Correspondence: James R Baker Jr Michigan Nanotechnology Institute, 4035 BSRB, 109 Zina Pitcher Place, SPC 2200, Ann Arbor, MI 48I 09-2200, USA

Tel +I 7346472777

Fax +I 7346152506

Email jbakerjr@umich.edu

\begin{abstract}
A target-specific MRI contrast agent for tumor cells expressing high affinity folate receptor was synthesized using generation five (G5) of polyamidoamine (PAMAM) dendrimer. Surface modified dendrimer was functionalized for targeting with folic acid (FA) and the remaining terminal primary amines of the dendrimer were conjugated with the bifunctional NCS-DOTA chelator that forms stable complexes with gadolinium (Gd III). Dendrimer-DOTA conjugates were then complexed with $\mathrm{GdCl}_{3}$, followed by ICP-OES as well as MRI measurement of their longitudinal relaxivity $\left(\mathrm{T} 1 \mathrm{~s}^{-1} \mathrm{mM}^{-1}\right)$ of water. In xenograft tumors established in immunodeficient (SCID) mice with KB human epithelial cancer cells expressing folate receptor (FAR), the 3D MRI results showed specific and statistically significant signal enhancement in tumors generated with targeted Gd(III)-DOTA-G5-FA compared with signal generated by non-targeted Gd(III)-DOTA-G5 contrast nanoparticle. The targeted dendrimer contrast nanoparticles infiltrated tumor and were retained in tumor cells up to 48 hours post-injection of targeted contrast nanoparticle. The presence of folic acid on the dendrimer resulted in specific delivery of the nanoparticle to tissues and xenograft tumor cells expressing folate receptor in vivo. We present the specificity of the dendrimer nanoparticles for targeted cancer imaging with the prolonged clearance time compared with the current clinically approved gadodiamide (Omniscan $\left.{ }^{\mathrm{TM}}\right)$ contrast agent. Potential application of this approach may include determination of the folate receptor status of tumors and monitoring of drug therapy.
\end{abstract}

Keywords: dendrimer, DOTA, gadolinium, MRI, folate receptor

\section{Introduction}

Clinical applications of magnetic resonance imaging (MRI) require generation of tissue-specific contrast. Numerous pulse sequences and methods have been designed to exploit signal differences created by endogenous contrast mechanisms such as T1, T2, the BOLD effect (Logothetis and Pfeuffer 2004), magnetization transfer (van Buchem and Tofts 2000), flow, and diffusion (Bammer et al 2005). Other imaging methods rely on injection or inhalation of contrast agents such as gadolinium $(\mathrm{Gd})$ chelates or hyperpolarized gases (Swanson et al 1997, 1999). Chelates of gadolinium ion such as Gd-DTPA generate contrast by increasing the relaxation rate of nearby water protons and are used in nearly half of all diagnostic MRI procedures (Shellock and Kanal 1999; Bellin 2006). These contrast agents are effective in diagnosis and staging of brain tumors by measuring the breakdown of the blood-brain barrier and in breast cancer by measuring enhanced uptake in breast tumors (Leach et al 2003). The contrast is generated by morphological changes to the tissue and not by targeting specific molecular aspects of the tumors. Indeed, specific targeting of cancer cells for imaging and treatment is thought to be a key component of cancer management in the near future and is currently an active area of research. Though a variety of molecular agents will likely play a role, the physical, chemical, 
and biological characteristics of branched polyamidoamide (PAMAM) dendrimers make this class of molecules an ideal platform for targeted therapeutic and contrast agents (Wiener et al 1994; Konda et al 2001; Konda et al 2002; Patri et al 2002; Kukowska-Latallo et al 2005; Venditto et al 2005).

PAMAM dendrimers are synthetic biocompatible macromolecules possessing multiple free amino groups on the surface. Generation-5 (G5) PAMAM dendrimers offer a carrier system having a defined branched structure capable of carrying multiple molecular entities, with a highly uniform size of about $5 \mathrm{~nm}$ in diameter (Kobayashi and Brechbiel 2005). The surface amino groups can be used for conjugation to molecules such as a dye or a drug to target cells in vitro and in vivo (Quintana et al 2002; Kukowska-Latallo et al 2005; Thomas et al 2005). PAMAM dendrimers are among the most promising of nanoparticle systems suggested recently because of their proven solubility in aqueous solutions, accessibility through the vasculature, lack of immunogenicity, and excretion through the kidney (Kukowska-Latallo et al 2005). The dendrimer platform allows synthesis of a nanometer size contrast particles (Kobayashi and Brechbiel 2005; Nasongkla et al 2006).

Targeting of the high affinity folate receptor for drug and contrast agent delivery provides one pathway for selective enhancement and/or treatment (Hilgenbrink and Low 2005). Initial studies by Konda et al using G4 dendrimers coupled with both folic acid (FA) and chelate (DTPA), and then complexed with gadolinium showed a promising targeted enhancement in ovarian tumor xenografts (Konda et al 2001). Therapeutic uses previously reported by us employed G5 PAMAM dendrimer conjugated with folic acid and methotrexate that specifically killed FA receptor-expressing human epithelial cancer cells (KB) by intracellular delivery of the drug through receptor-mediated endocytosis in vitro and in vivo (Kukowska-Latallo et al 2005; Patri et al 2005).

In this study, we present the pharmacokinetics and tissue distribution of G5 PAMAM dendrimer conjugated with folic acid and DOTA-NCS, and complexed with gadolinium (Gd III) in a murine model of human cancer. To control for nonspecific uptake of dendrimer, both targeted and non-targeted contrast agents were synthesized and studied. We report specific binding of the folic acid targeted contrast agents to folate receptor expressing tumor cells in vivo.

\section{Materials and methods}

\section{Synthesis of dendrimer-gadolinium nanoparticle}

The PAMAM generation 5 (G5) dendrimers were synthesized at the Michigan Nanotechnology Institute for Medicine and Biological Sciences. Folic acid (FA), acetic anhydride, triethylamine, 1-ethyl-3-(3-dimethylaminopropyl) carbodiimide hydrochloride (EDC) and all the solvents were purchased from Sigma-Aldrich (USA). The bifunctional chelating agent DOTA-NCS $(1,4,7,10$-tetraazacyclododecane-1,4,7,10-tetraacetic acid, $\alpha$-[5-isothiocyanato-2methoxyphenyl]-, hydrochloride [9Cl]) was provided by Dow Chemical Company (Midland, MI, USA).

Two contrast nanoparticles were synthesized and evaluated; targeted Gd(III)-DOTA-G5-FA contrast agent and a non-targeted Gd(III)-DOTA-G5. The G5 PAMAM dendrimer was first recovered from concentrated G5/ methanol solution by the removal of methanol and lyophilization in water. For targeting experiments G5 dendrimer was conjugated with an average of 4.5 folic acid molecules conjugated to free amines. In partial acetylation that was carried out to improve the solubility of the final dendrimer-Gd complex 50 of 110 primary amine groups of the dendrimer were acetylated as shown in schematic synthesis of dendritic contrast agents (Scheme 1). The remaining terminal primary amines of the dendrimer were conjugated with the bifunctional DOTA-NCS. The control G5 PAMAM dendrimer was completely surface functionalized with DOTA-NCS chelator without targeting folic acid moiety on the surface. The isothiocyanate reaction was run at $\mathrm{pH} 9$ to generate free amines at a molar ratio of DOTA to amine equal $2: 1$. The dendritic chelator was then complexed with $30 \%$ molar excess of $\mathrm{GdCl}_{3} \cdot 6 \mathrm{H}_{2} \mathrm{O}$ at $\mathrm{pH} 6$ adjusted with citrate buffer. The reaction was performed at room temperature and was completed in 48 hours. The conjugates were purified in amicon stirred cell with $10 \mathrm{~K}$ MWCO membrane. The dendrimers conjugated with chelators (without Gd) were characterized by NMR, HPLC and GPC. Dendritic chelators were titrated with $\mathrm{GdCl}_{3}$ and followed by ICP-OES spectroscopy as well as their longitudinal relaxation times (T1) of water. The absence of free Gd(III) was confirmed by comparing UVVis spectra of G5 dendrimer conjugate complexed with Gd before and after addition of photometric Arsenazo III agent (Nagaraja et al 2006). The Gd(III) concentration of the contrast agents was quantified by inductive coupled plasma-optical emission spectrometry (ICP-OES). Analysis revealed that targeted imaging agent [Gd(III)-DOTA-G5FA] was loaded with $6.0 \%$ of Gd(III) and the non-targeted contrast agent [Gd(III)-DOTA-G5] was loaded with 7.7\% of $\mathrm{Gd}$. Both compounds were dissolved in sterile saline to equimolar concentrations of $\mathrm{Gd}(\mathrm{III})$ at $0.45 \mathrm{mg} / \mathrm{mL}$ and $0.9 \mathrm{mg} / \mathrm{mL}$. 


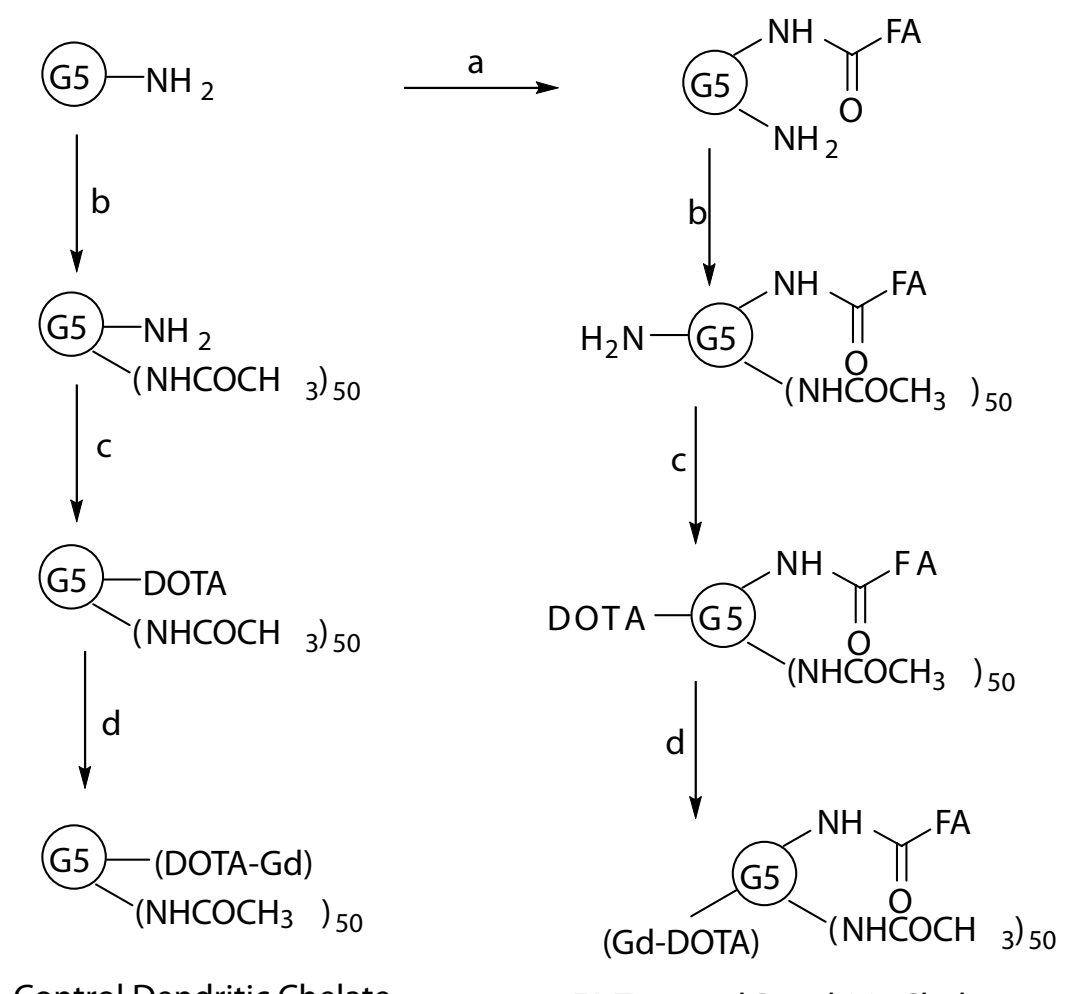

Control Dendritic Chelate

FA Targeted Dendritic Chelate

a) Folicacid/DMSO/EDC; b) Acetic anhydride (50x)/DMS O; c) NCS-DOTA , pH 9.0; d) GdCl3, pH 6.0.<smiles>COc1ccc(N=C=S)cc1C(C(=O)O)N1CCN(CC(=O)O)CCN(CC(=O)O)CCN(CC(=O)O)CC1</smiles>

Scheme I The synthesis of control (Gd[III]-DOTA-G5) and FA targeted dendritic chelate (Gd[III]-DOTA-G5-FA).After conjugation of 4.5 (on average) of folic acid molecules to G5 PAMAM dendrimer (a), 50 of the primary amine groups were acetylated (b), the remaining primary amines conjugated with bifunctional NCS-DOTA (c) and complexed with $\mathrm{GdCl}_{3} \cdot 6 \mathrm{H}_{2} \mathrm{O}(\mathbf{d})$. The structure of the DOTA-NCS used for conjugation of contrast agents (lower panel).

\section{$M R$ imaging in vitro}

The KB human cell line over-expresses folate receptor (FAR) when grown in folate-deficient medium (Kukowska-Latallo et al 2005). Cells were purchased from the American Type Culture Collection (ATCC, Manassas, VA, USA) and maintained at $37{ }^{\circ} \mathrm{C}$ with $5 \% \mathrm{CO}_{2}$ in folate-deficient RPMI 1640 supplemented with penicillin (100 units $/ \mathrm{mL}$ ), streptomycin $(100 \mu \mathrm{g} / \mathrm{mL})$, and $10 \%$ heat-inactivated FBS. Cell suspensions created with $5 \times 10^{6} \mathrm{~KB}$ cells in PBS (phosphate-buffered saline) were incubated with targeted and non-targeted contrast agents at $0.01 \mathrm{mM}$ concentration of $\mathrm{Gd}(\mathrm{III})$ at $4{ }^{\circ} \mathrm{C}$ for $30 \mathrm{~min}$.
Following incubation cells were washed 3 times with PBS and centrifuged each time at $500 \times \mathrm{g}$ for $5 \mathrm{~min}$. After removal of supernatant the volume of cell pellets was approximately $100 \mu \mathrm{L}$. The relaxation rates of labeled cells in wet pellets were measured at $2 \mathrm{~T}$ and at $20^{\circ} \mathrm{C}$.

\section{Recipient animal and tumor model}

A murine tumor model was established in NOD C.B-17 SCID mice using human KB tumor cells over-expressing folate receptor as described previously (Kukowska-Latallo et al 2005). Briefly, 5- to 6-week-old FOX CHASE 
SCID (C.B-17/lcrCrl-scidBR) female mice were purchased from the Charles River Laboratories (Wilmington, MA, USA) and housed in a specific pathogen-free animal facility at the University of Michigan Health System in accordance with the regulations of the University's Committee on the Use and Care of Animals (UCUCA) as well as with federal guidelines, including the Principles of Laboratory Animal Care. Animals were fed ad libitum with Laboratory Autoclavable Rodent Diet 5010 (PMI Nutrition International, St. Louis, MO, USA). The food was changed to a folate-deficient diet (TestDiet, Richmond, IN, USA) for 7 days prior to injection of tumor KB cells on both flanks of mice. Normal rodent chow contains a high concentration of folic acid $(6 \mathrm{mg} / \mathrm{kg}$ chow) and on this diet the mouse serum folate concentration is about 50 times higher than human. Using the low folate diet brings down mouse serum levels to about 2 times the folate concentration in normal human serum (Mathias et al 1998). The FR receptor in the tumor is not altered due to this dietary intervention (Kukowska-Latallo et al 2005). Subcutaneous KB tumor nodules appeared 7-10 days post-implantation. Anesthesia for imaging was induced by placing mice in a chamber with $2.0 \%$ isofluroane (SBH Scientific). Following anesthetic induction, the mice were transferred to the MRI probe and anesthesia was maintained at $1.25 \%$ isoflurane for the duration of the study. All procedures were performed under protocol approval by UCUCA at the University of Michigan.

\section{Delivery of $\mathrm{Gd}(\mathrm{III})$ contrast nanoparticle}

Imaging agent was delivered through intravenous injection at 20 or 30 days post-implantation. The Gd(III)-DOTAG5-FA and the non-targeted Gd(III)-DOTA-G5 dendrimer nanoparticles were delivered via the tail vein in $0.2 \mathrm{~mL}$ of saline at $0.029 \mathrm{mmol}$ of $\mathrm{Gd} / \mathrm{kg}$ and at $0.071 \mathrm{mmol} / \mathrm{kg}$. Two-dimensional and three-dimensional MRI images were obtained both before and after administration of either imaging agent at 1, 4, 24, 48, and 96 hours post injection.

\section{MR imaging in vivo}

The MRI probe, constructed specifically for these studies, was based on an Alderman-Grant slotted cylinder design (length 10 $\mathrm{cm}, \mathrm{OD} 4.5 \mathrm{~cm}$ ). The probe was made with polycarbonate tubing, copper tape, and ATC and Johanson capacitors. Following induced anesthesia, the mouse to be imaged was placed inside a second polycarbonate tube (ID $2.6 \mathrm{~cm}$ ). This second tube was then inserted into the MR probe, allowing easy animal positioning and restricting mouse MR studies to a region of homogeneous RF field. MRI was performed on a 2T Varian Unity/Inova system equipped with Acustar S180 gradients.

The 2D and 3D gradient-echo MR images were obtained at each time point for each animal. Two sets of interleaved, 2D gradient-echo images were acquired with $2 \mathrm{~mm}$ slice thickness, TR/TE $100 / 5 \mathrm{~ms}$, flip angle $45^{\circ}$, in plane resolution $390 \mu \mathrm{m}$, and 8 averages. Total time to acquire the $2 \mathrm{D}$ images was 2.5 minutes. The $3 \mathrm{D}$ gradient-echo images were acquired with a TR/TE of $20 / 4 \mathrm{~ms}$, a flip angle of $20^{\circ}$, isotropic voxel resolution of $390 \mu \mathrm{m}$, and 4 averages. Imaging time for the $3 \mathrm{D}$ dataset was $5.5 \mathrm{~min}$. The $3 \mathrm{D}$ gradient echo pulse sequence was chosen to provide isotropic spatial resolution, minimize motion artifacts, and generate T1 weighted MR images.

All image data were transferred to a remote computer (Dual $2 \mathrm{GHz}$ PowerPC G5 Macintosh) for data processing. Matlab (The Mathworks, Natick, MA, USA) was used to convert the $\mathrm{k}$-space MR data sets into quantitative MR images and to combine all images of the study into a single dataset. Tumor was typically present in 30 of 64 planes in the 3D image. The entire tumor was selected from the dataset and used to generate normalized histograms of the signal intensity. Using the data from 3D MR studies assures that the entire tissue of interest is examined and is not biased toward anomalously enhancing regions. The percentage enhancement was calculated for each of the images acquired at each time following injection of either the targeted or the non-targeted contrast agent. The normalized histogram of the percentage signal enhancement was created by defining the sum of all signals to $100 \%$. Histograms in the figures are plotted as the percentage of voxels at a given percentage enhancement.

\section{Statistical methods}

To test the hypothesis that targeted contrast agent generates statistically significant signal enhancement, a 2-sample Kolmogorov-Smirnov (Massey 1951) (KS) test was used (Matlab, Natick, MA, USA). The KS test was applied to the normalized histograms of the signal intensities of the MR data from targeted and non-targeted contrast agent studies in tumor and other tissues. This statistic was chosen because the distribution of signal intensity generated by the contrast agent is not necessarily normal and because the large number of voxel intensities generates a well sampled distribution. P-values of less that 0.05 allow us to reject the null hypothesis that the two samples are drawn from the same underlying population.

\section{Results}

The relaxivity of the contrast agents measured in solutions at $2 \mathrm{~T}$ and at $20^{\circ} \mathrm{C}$ was $26.0 \pm 0.1 \mathrm{~s}^{-1} \mathrm{mM}^{-1}$ for targeted 
Gd(III)-DOTA-G5-FA agent and $19.4 \pm 0.04 \mathrm{~s}^{-1} \mathrm{mM}^{-1}$ for the non-targeted Gd(III)-DOTA-G5 agent. The calculated relaxivity of the milimole of the targeted and non-targeted contrast nanoparticle is $598 \mathrm{~s}^{-1} \mathrm{mM}^{-1}$ and $532 \mathrm{~s}^{-1} \mathrm{mM}^{-1}$, respectively. In comparison gadolinium complex of diethylenetriamine pentaacetic acid bismethylamide used as clinical contrast (Gd(III)-DTPA-BMA; Omniscan ${ }^{\mathrm{TM}}$ ), has a relaxivity of $3.9 \mathrm{~s}^{-1} \mathrm{mM}^{-1}$ in solution (Bellin 2006). The relaxivity of $\mathrm{Gd}$ chelates bonded to these dendrimers is more than 4 times greater than Omniscan ${ }^{\mathrm{TM}}$. The increased relaxivity is due to a decrease of the rotational correlation time of the Gddendrimer complex (Bryant et al 1999; Nicolle et al 2002; Laus et al 2005; Rudovsky et al 2006). For contrast agents such as these molecules, increased relaxivity is one of the most important measures of the effectiveness of the contrast agent. Therefore, the increased relaxivity is a very desirable property of dendrimer contrast agents.

Targeting specificity was measured in vitro by incubating $\mathrm{KB}$ tumor cells over-expressing folate receptor to targeted and non-targeted contrast agents at $0.01 \mathrm{mM}$ concentration of Gd(III). Relaxation rate $\mathrm{R} 1$ of wet cell pellets after incubation of cells with each contrast agent was measured in $2.0 \mathrm{~T}$ MR system and at $20^{\circ} \mathrm{C}$, and was compared with untreated cells. The targeted contrast agent specifically binds to $\mathrm{KB}$ tumor cells and increases the water proton relaxation rate whereas the non-targeted contrast agent creates no additional relaxation when compared to the relaxation rate of untreated cells (Table 1).

The in vivo MRI results using dendrimer nanoparticles are presented on one slice of the $3 \mathrm{D}$ dataset from the images acquired following injection of either targeted Gd(III)-DOTA-G5-FA or non-targeted Gd(III)-DOTA-G5 (Figure 1). The image has been colorized to allow easy visualization of the contrast changes as a function of time post-injection. The control image was obtained from mouse injected with saline (Figure 1a). The animals injected with

Table I Relaxation rates $(\mathrm{RI}) \pm$ estimated error of inversion recovery fit of $K B$ cells labeled with non-targeted and targeted (FA) Gd loaded $\mathrm{G} 5$ dendrimers, both at adjusted $0.01 \mathrm{mM}$ concentration of $\mathrm{Gd}$, were measured in wet cell pellets. Increased relaxation rate of cells labeled with the targeted contrast agent indicates specific binding to KB cells. Cell pellets were created with $5 \times 10^{6}$ cells and had an approximate volume of $100 \mu \mathrm{L}$. All relaxation rates were measured at $2 \mathrm{~T}$ and at $20^{\circ} \mathrm{C}$

\begin{tabular}{ll}
\hline Compound & RI (s-1) \\
\hline Gd(III)-DOTA-G5 & $0.479 \pm 0.013$ \\
Gd(III)-DOTA-G5-FA & $0.877 \pm 0.001$ \\
none & $0.484 \pm 0.001$ \\
\hline
\end{tabular}

FAR-targeted contrast agent presented increased signal intensity in liver, kidney, gut, and tumor (Figure 1a). Data from tumor were selected and used to create the normalized histograms of signal enhancement for all time points post-injection (Figure $1 \mathrm{~b}$ and $1 \mathrm{c}$ ). Due to the presence of the high-molecular weight contrast agent there is already significant signal enhancement at 1 hour (red line) that remains at the same level at 4 hours (green line) with slow decrease starting at 24 hours (blue line) and continuing at 48 hours (black) (Figure 1b). These data show increase in both the average signal enhancement and the spread of the signal enhancement. The non-specific enhancement of non-targeted Gd(III)-DOTA-G5 contrast is present only at 1 hour (red line), prior to being cleared by the kidney (Figure 1c). This clearance is supported by very bright signal intensity of water in the bladder obtained for the 1 hour and 4 hour images (data not shown). Statistical analysis (KS test) performed between the time matched histograms of the targeted tumor (Figure 1b) and histograms of nontargeted tumor (Figure 1c) showed statistically significant differences at 1, 4, and 24 hours (Table 2).

Data selected from liver, kidney and muscle tissue were reduced to normalized histogram of signal enhancements as described above. The averages $\pm \mathrm{SD}$ of the percent signal enhancement is shown for tumor, kidney, liver, and muscle, for both targeted and non-targeted contrast agent and for all times post-injection (Figure 2a, b, c, d). In addition to the tumor enhancement, significant signal enhancement for the targeted contrast agent study is observed for kidney and liver tissues at 1 and 4 hours post injection (Figure 2b, c). Enhancement in kidney and liver is expected due to expression of folic acid receptors in these tissues (Figure 2b and 2c) (Parker et al 2005). Muscle, with no known expression of folate receptor, does not show significant enhancement for either the targeted or the non-targeted contrast agent (Figure 2d) (Parker et al 2005).

An additional study of the differential enhancement of tumors with targeted and non-targeted Gd(III)-DOTA-G5 was performed using higher dose of contrast $(0.072 \mathrm{mmol} / \mathrm{kg})$, comparable and recommended for clinical gadolinium contrast agent, Omniscan $^{\mathrm{TM}}$ (Figure 3a, b, c, d). Targeted compound generates further increased signal enhancement when compared to the results of Gd(III)-DOTA-G5-FA in Figure 1. The most significant difference was observed at 4 hour post-injection showing no overlap between histograms for targeted and non-targeted contrast nanoparticles (Figure $3 \mathrm{a}, \mathrm{b}$ ). The signal is maintained for up to 48 hours (Figure 3c) compared with 24 hours in the earlier study 


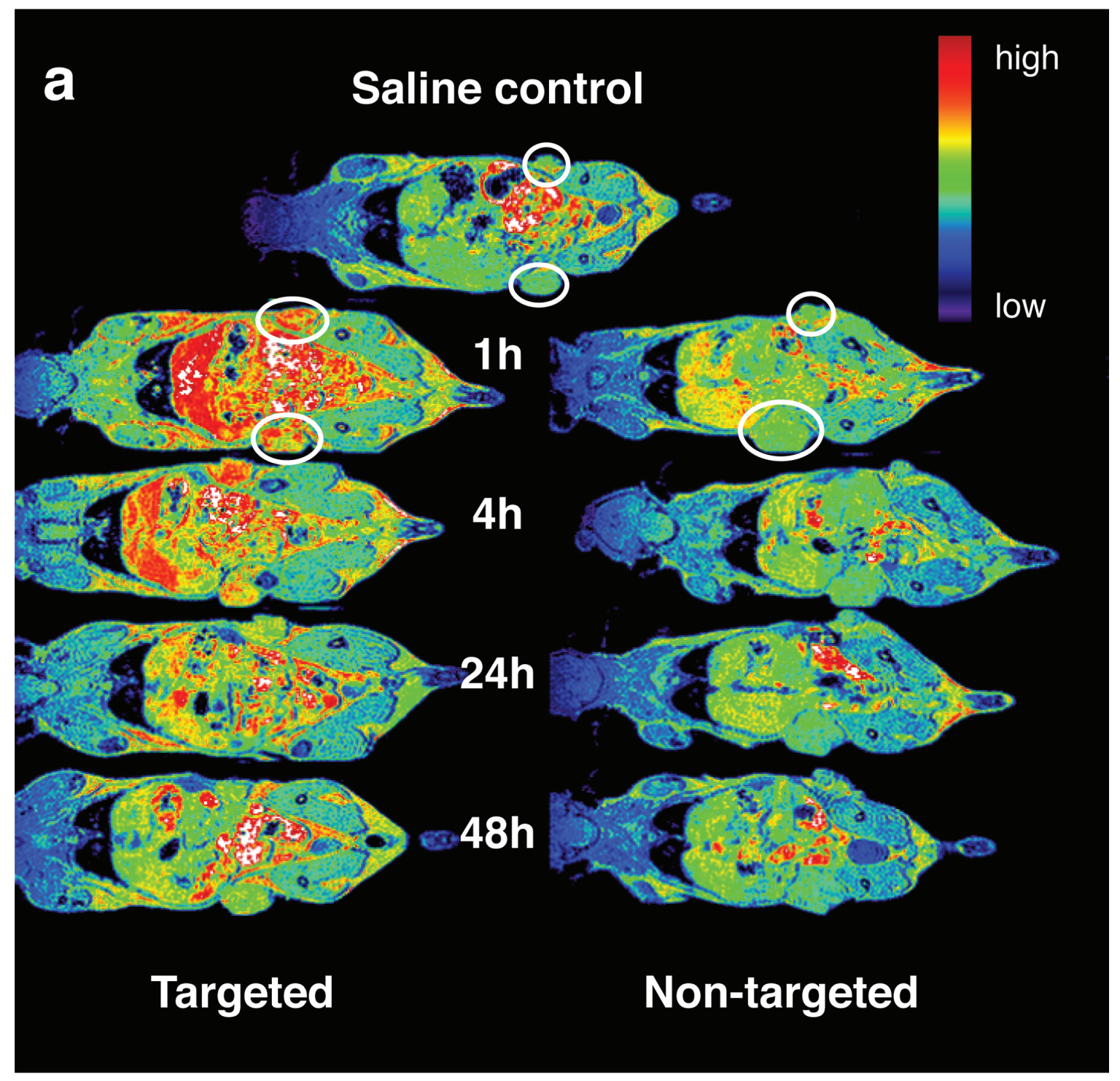

b

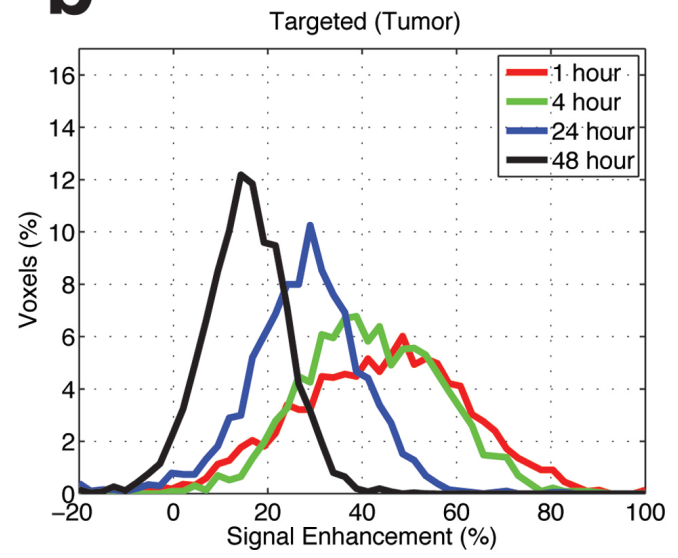

C

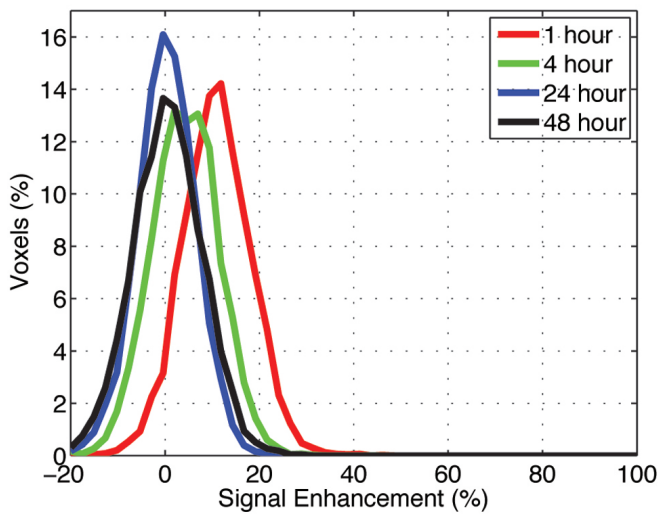

Figure I MR imaged uptake of targeted and non-targeted contrast nanoparticles delivered at $0.029 \mathrm{mmol} / \mathrm{kg}$ of Gd. The top image is of a control mouse injected with saline and imaged at Ihour (a). The series of images on the left from the mouse injected with targeted contrast Gd(III)-DOTA-G5-FA and on the right from the mouse injected with the non-targeted contrast agent Gd(III)-DOTA-G5 were collected at I, 4, 24, and 48 hours post-injection (a). The circles are drawn around the tumor to guide the eye. Signal enhancement is created by increased water proton relaxation due to the presence of the contrast agent. Normalized histograms of the voxel intensities were created for the whole tumors on both flanks of mouse, for the two mice injected with targeted and non-targeted contrast presented on the series of images (a). Comparison of targeted (b) and non-targeted contrast (c) showed statistically significant difference (Table 2; KS test, p $<0.05$ ) between the signals collected at I, 4 , and 24 hours. 
Table $2 \mathrm{P}$ values of $\mathrm{KS}$ test between normalized histograms of signal intensities of the MR data from Gd(III)-DOTA-G5-FA and Gd(III)DOTA-G5 contrast agents in tumors shown in Figure $\mathrm{Ib}$ and Ic. Histograms at I, 4, and 24 hours show a statistically significant difference. $P$ values of less than 0.05 allow us to reject the null hypothesis that the two samples are drawn from the same underlying population

\begin{tabular}{ll}
\hline Time (hours) & P value KS test \\
\hline $\mathrm{I}$ & $=0.0000 \mathrm{I}$ \\
4 & $=0.00096$ \\
24 & $=0.00017$ \\
48 & $=0.50(\mathrm{~ns})$ \\
\hline
\end{tabular}

(Figure 1c). The increased signal intensities due to higher concentration of contrast give more efficient in vivo relaxation and stronger saturation of the folate receptor. The difference in the spreads of the distributions between non-targeted contrast agents (Figure 3c and 3d) can be further explained by the fact that the first study was begun 20 days following tumor implantation (Figure 1) and the second one was started 30 days after implantation (Figure 3 ). The larger size of tumors could account for a broader signal intensity distribution.

To control for non-specific uptake for targeted-dendrimer nanoparticle an additional study was performed using a mouse with different xenograft tumors on each flank, one side with KB cells expressing FAR and the other with MCA207 cells negative for FAR (Figure 4). The signal enhancement was observed in KB tumors positive for FAR when folate-targeted dendrimer contrast was delivered (Figure 4, right panel, A). Some capture was observed for MCA207 tumor, but largely to the same extent as of non-targeted dendrimer contrast in both MCA207 and KB tumors (Figure 4, right panel, B).

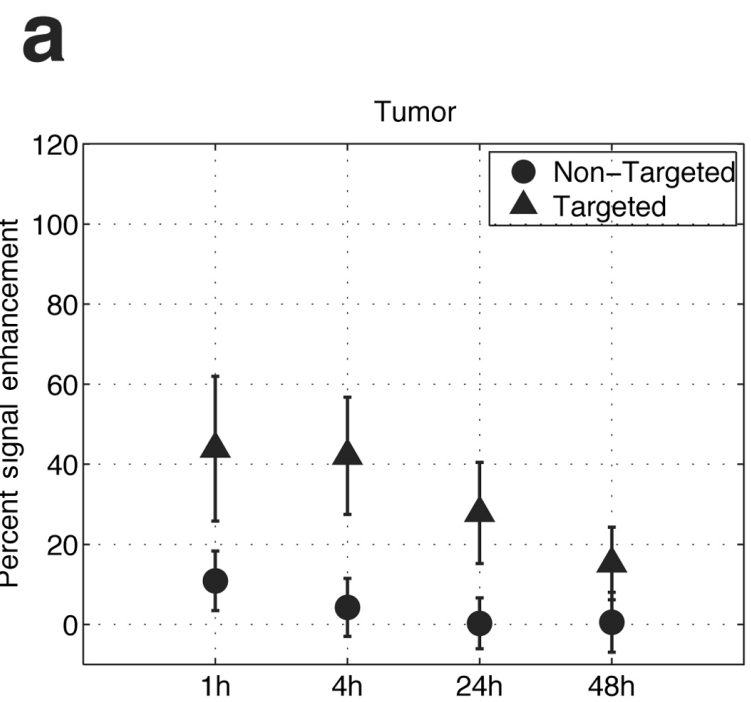

b
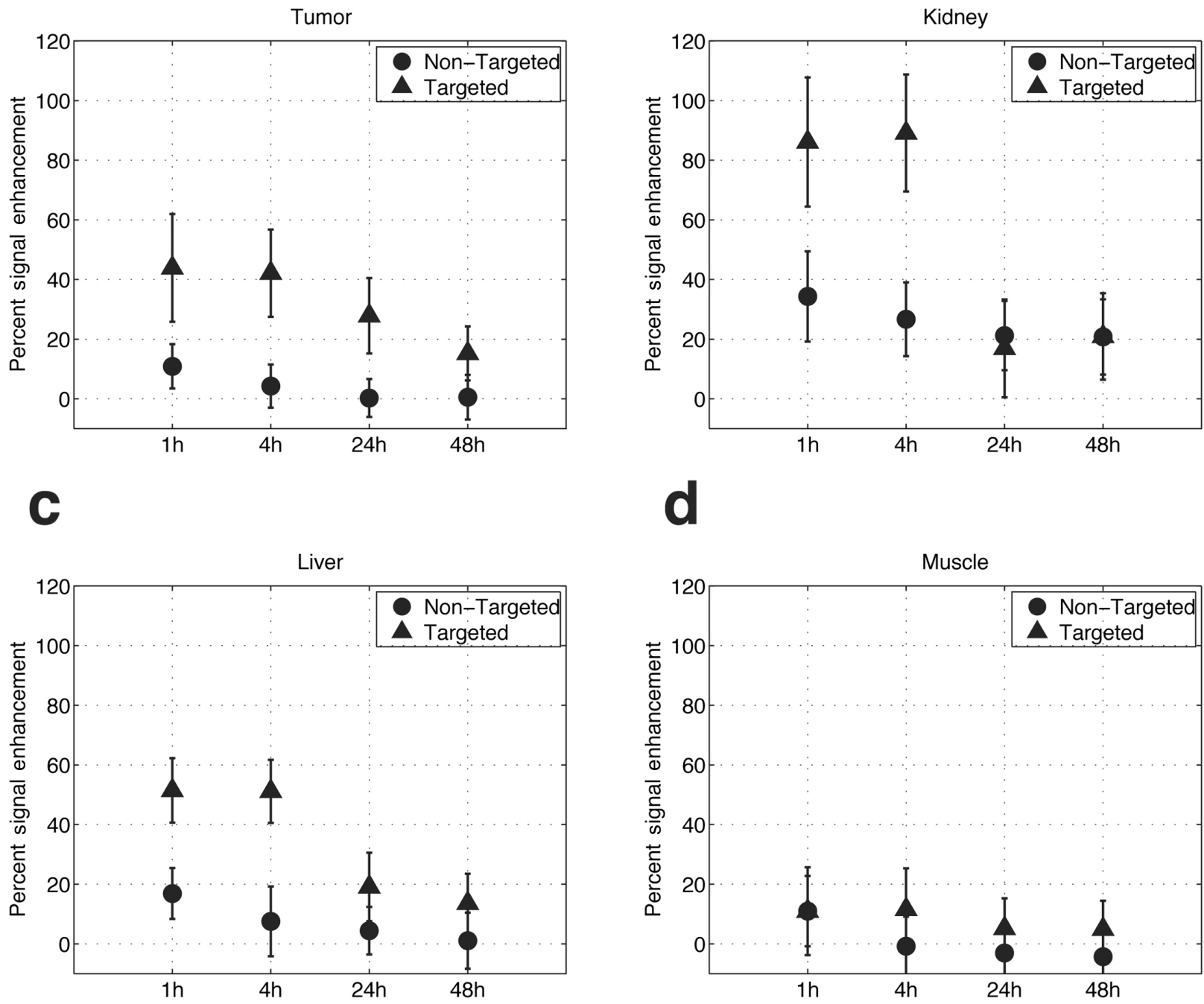

Figure 2 Signal enhancement in tumor (a), kidney (b), liver (c) and, muscle (d) in the two mice injected with targeted [Gd(III)-DOTA-G5-FA] or non-targeted [Gd(III)-DOTA-G5] contrast presented in Figure Ia. A significant difference between signal enhancement of the two contrast agents is observed at I,4, and 24 hours for the tumor (a) and at I and 4 hours for the kidney (b) and the liver (c) indicating preferential uptake of targeted contrast agent. Signal in muscle shows no enhancement for either contrast agent (d). 
a b

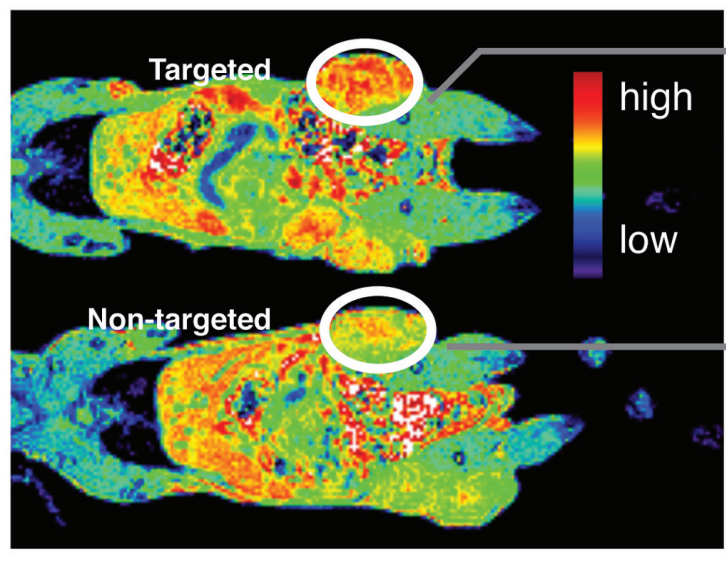

Targeted vs. Non-targeted at $4 \mathrm{~h}$

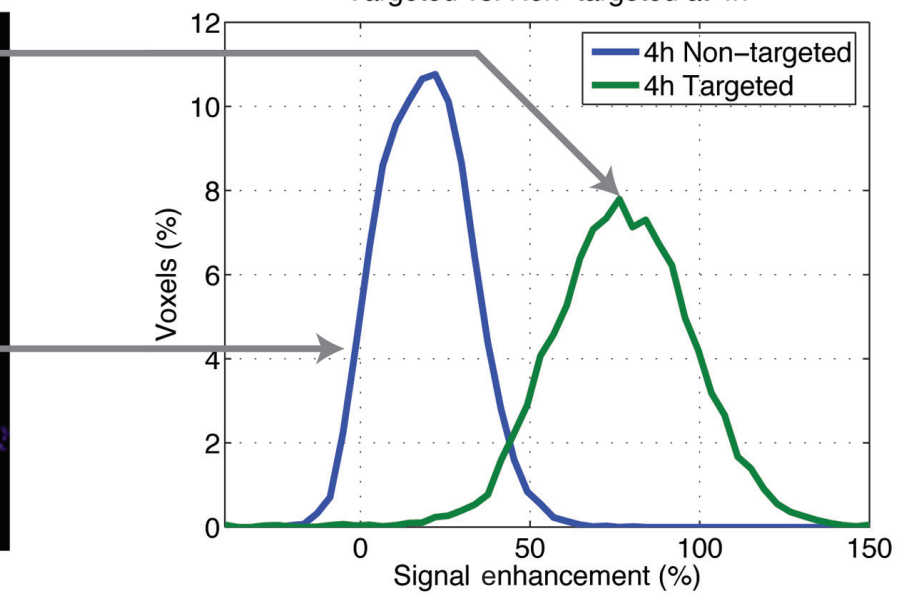

C

Targeted

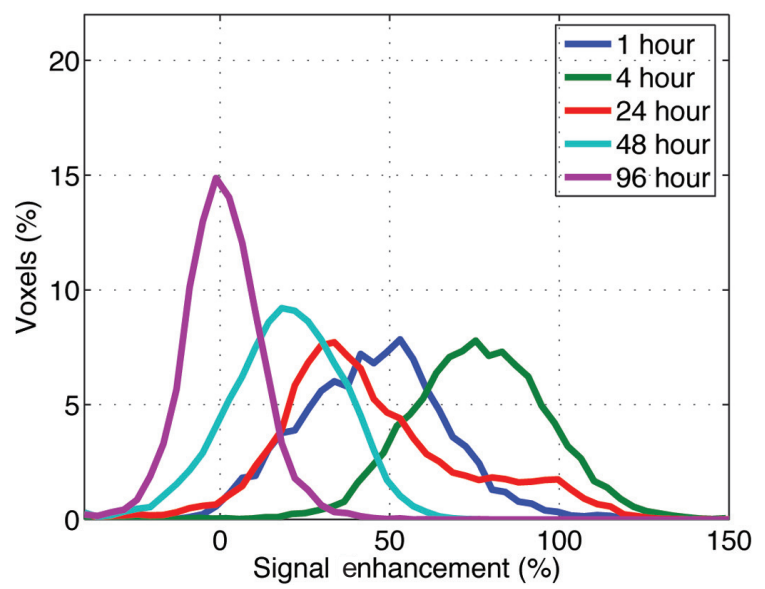

d

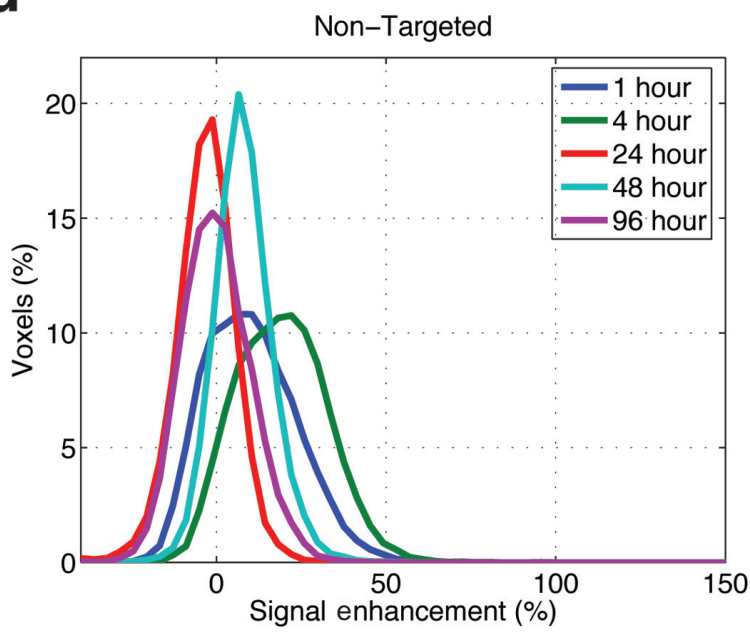

Figure 3 MR images obtained at a higher dose of Gd $(0.072 \mathrm{mmol} / \mathrm{kg})$ of targeted Gd(III)-DOTA-G5-FA and non-targeted Gd(III)-DOTA-G5 contrast nanoparticles (a). A significant difference in the signal intensity is observed at 4 hours (b) and from I to 24 hours post-injection of contrast nanoparticles (c, d). The signal persists up to 48 hours post-injection (c) with a minimal overlap between targeted and non-targeted contrast agents up to 24 hours (c, d).

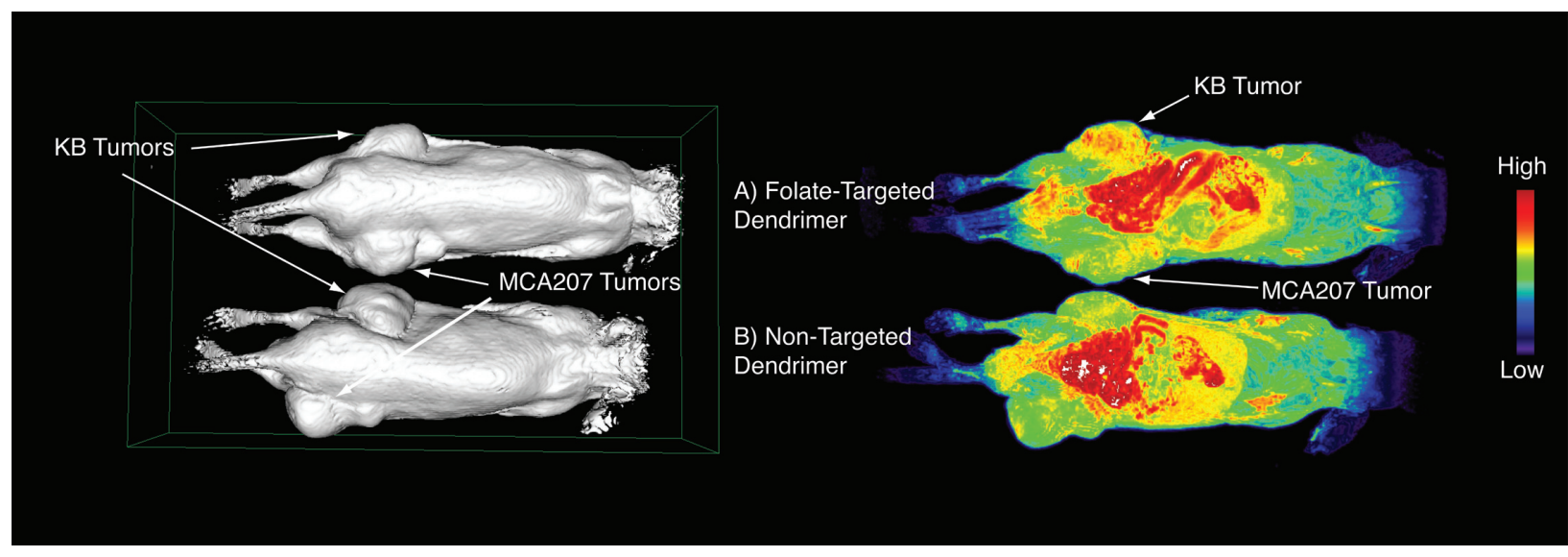

Figure 4 Surface rendering (left panel) and signal enhancement images (right panel) of the same pair of mice bearing KB tumors (upper flank of mouse) and MCA207 tumors (lower flank of mouse) injected with folate-targeted contrast nanoparticle (A) and non-targeted contrast nanoparticle (B). The KB tumor that expresses FAR showed signal enhancement at 24 hours post-injection of targeted-contrast (right panel, A, upper flank) compared with some capture in MCA207 tumor, which was non-specific for both targeted and non-targeted contrast nanoparticles. 


\section{Discussion}

Improvement of the diagnostic specificity of MRI imaging can be achieved through application of different types of imaging probes that provide high-resolution anatomical information and functional measurement of tumor physiology (Nasongkla et al 2006; Talanov et al 2006). MRI contrast probes containing a paramagnetic cation or nanosized particle can be classified as non-specific, targeted or activatable (Mathias et al 2003). Targeting of imaging agents is currently a vibrant area of active research. Specific localization to defined cells via internalization or interaction with cell surface proteins of target cell can result in tumor-specific accumulation and enhanced detection of tumor burden in vivo (Kobayashi et al 2004; Nasongkla et al 2006). The increased retention of targeted probes compared with rapidly diffusing Gd(III) chelates used for clinical MRI examinations allows for longer time of detection due to increased stability in vivo (Figures 1-3). Taken together, our results indicate that targeted MR nanoparticles can be used to follow the administration of different therapeutic agents, thereby contributing to the effectiveness of a drug. The technique may be used to screen cancers for receptor status and to follow up drug therapy in tumors responding to therapy. The targeted folatedendrimer nanoparticles were taken up heavily in the liver, bowel and kidney due to excretion of the conjugates and the presence of folate receptors in these tissues. The possible clinical targets for the imaging technique presented here could be ovarian, breast, and head and neck tumors (Kalli et al 2008). Dendrimer-based delivery of contrast has several additional advantages including small size ( $\sim 30 \mathrm{kD})$ comparable to size of proteins, that allows escape from vasculature and target tumor cells while also being below the threshold of renal filtration and therefore removing a requirement for hepatic metabolism. The G5 dendrimer is stable, provides stability for Gd chelates, is non-immunogenic, and contains ample reactive sites for the conjugation of complex drug delivery systems (Kukowska-Latallo et al 2005).

Our study uses the dendrimer-gadolinium nanoparticles as a $\mathrm{T} 1$ contrast agent. The experiments were performed with $\mathrm{G} 5$ dendrimer at $2.0 \mathrm{~T}$. At $2.0 \mathrm{~T}$, the high molecular weight slows the rotation of the G5 Gd-dendrimer complex and tunes the electron-nuclear dipole-dipole correlation time to the proton Larmor frequency; an ideal situation for efficient spin-lattice (T1) relaxation. This mechanism is operative only from about 0.5 to $3.0 \mathrm{~T}$. At these field strengths, T1 weighted pulse sequences can be used and the presence of the contrast agent indicated by an increase in the signal intensity. Because T1 contrast agents create signal enhancement on $\mathrm{T} 1$ weighted pulse sequence, they are generally thought to be more desirable than $\mathrm{T} 2$ contrast agents that create signal loss. Furthermore, future clinical applications of these contrast agents are likely to be performed at either 1.5 or $3.0 \mathrm{~T}$.

In summary we have demonstrated a target-specific MRI contrast agent for tumor cells expressing high affinity folate receptor. The Gd(III)-DOTA-G5-FA contrast nanoparticle specifically binds to xenograft tumors established with human epithelial cancer cells (KB) which over-express folate receptor and generates statistically significant signal enhancement in tumors when compared to signal enhancement generated by non-targeted Gd(III)-DOTA-G5 contrast agent. The specific enhancement observed in liver and kidney is due to presence of folate receptor in these tissues (Parker et al 2005). We have used 3D MR data to examine signals from the entire tumor or organ and the KS statistical test to establish differences in the histograms of targeted versus non-targeted contrast nanoparticles. We report a controlled, statistically significant and tissue specific increase in MR signal intensity in tumors for targeted dendrimer nanoparticles compared with the non-targeted nanoparticles. While we believe that based on this approach translational research may be possible, this clearly will require more complete GLP toxicity studies that are beyond the scope of the current work.

\section{Acknowledgments}

This work was supported in part by National Cancer Institute (NCI), NIH contract N01-CO-97111, NCI-NIH (1 R01 EB002657), Michigan Economic Development CorporationLife Sciences Corridor Fund (GR-472) to JRB.

Drs. S D Swanson and J F Kukowska-Latallo contributed equally to this work.

Dr. Kevin McDonagh (University of Kentucky) is gratefully acknowledged for the donation of the MCA207 cells.

\section{Disclosures}

The University of Michigan patented research based on the discoveries cited in this paper. In addition, this technology is licensed to a startup company, Avidimer Therapeutics, in which both the University of Michigan and Dr. Baker have a significant financial interest.

\section{References}

Bammer R, Skare S, Newbould R, et al. 2005. Foundations of advanced magnetic resonance imaging. NeuroRx, 2:167-96.

Bellin MF. 2006. MR contrast agents, the old and the new. Eur J Radiol, $60: 314-23$. 
Bryant LH Jr, Brechbiel MW, Wu C, et al. 1999. Synthesis and relaxometry of high-generation $(\mathrm{G}=5,7,9$, and 10) PAMAM dendrimer-DOTAgadolinium chelates. J Magn Reson Imaging, 9:348-52.

Hilgenbrink AR, Low PS. 2005. Folate receptor-mediated drug targeting: from therapeutics to diagnostics. $J$ Pharm Sci, 94:2135-46.

Kalli KR, Oberg AL, Keeney GL, et al. 2008. Folate receptor alpha as a tumor target in epithelial ovarian cancer. Gynecol Oncol, 108:619-26

Kobayashi H, Brechbiel MW. 2005. Nano-sized MRI contrast agents with dendrimer cores. Adv Drug Deliv Rev, 57:2271-86.

Kobayashi H, Kawamoto S, Sakai Y, et al. 2004. Lymphatic drainage imaging of breast cancer in mice by micro-magnetic resonance lymphangiography using a nano-size paramagnetic contrast agent. $J$ Nal Cancer Inst, 96:703-8.

Konda SD, Aref M, Wang S, et al. 2001. Specific targeting of folatedendrimer MRI contrast agents to the high affinity folate receptor expressed in ovarian tumor xenografts. Magma, 12:104-13.

Konda SD, Wang S, Brechbiel M, et al. 2002. Biodistribution of a $153 \mathrm{Gd}-$ folate dendrimer, generation $=4$, in mice with folate-receptor positive and negative ovarian tumor xenografts. Invest Radiol, 37:199-204.

Kukowska-Latallo JF, Candido KA, Cao Z, et al. 2005. Nanoparticle targeting of anticancer drug improves therapeutic response in animal model of human epithelial cancer. Cancer Res, 65:5317-24.

Laus S, Sour A, Ruloff R, et al. 2005. Rotational dynamics account for $\mathrm{pH}$-dependent relaxivities of PAMAM dendrimeric, Gd-based potential MRI contrast agents. Chemistry, 11:3064-76.

Leach MO, Brindle KM, Evelhoch JL, et al. 2003. Assessment of antiangiogenic and antivascular therapeutics using MRI: recommendations for appropriate methodology for clinical trials. Br J Radiol, 76(Spec No 1):S87-91.

Logothetis NK, Pfeuffer J. 2004. On the nature of the BOLD fMRI contrast mechanism. Magn Reson Imaging, 22:1517-31.

Massey FJ. 1951. The Kolmogorov-Smirnov Test for Goodness of Fit. J Am Stat Assoc, 46:68-78.

Mathias CJ, Lewis MR, Reichert DE, et al. 2003. Preparation of Ga-66- and Ga-68-labeled Ga(III)-deferoxamine-folate as potential folate-receptortargeted PET radiopharmaceuticals. Nucl Med Biol, 30:725-31.

Mathias CJ, Wang S, Waters DJ, et al. 1998. Indium-111-DTPA-folate as a potential folate-receptor-targeted radiopharmaceutical. $\mathrm{J} \mathrm{Nucl} \mathrm{Med}$, 39:1579-85.

Nagaraja TN, Croxen RL, Panda S, et al. 2006. Application of arsenazo III in the preparation and characterization of an albumin-linked, gadoliniumbased macromolecular magnetic resonance contrast agent. $J$ Neurosci Methods, 157:238-45.
Nasongkla N, Bey E, Ren JM, et al. 2006. Multifunctional polymeric micelles as cancer-targeted, MRI-ultrasensitive drug delivery systems. Nano Letters, 6:2427-30.

Nicolle GM, Toth E, Schmitt-Willich H, et al. 2002. The impact of rigidity and water exchange on the relaxivity of a dendritic MRI contrast agent. Chemistry, 8:1040-8.

Parker N, Turk MJ, Westrick E, et al. 2005. Folate receptor expression in carcinomas and normal tissues determined by a quantitative radioligand binding assay. Anal Biochem, 338:284-93.

Patri AK, Kukowska-Latallo JF, Baker JR Jr. 2005. Targeted drug delivery with dendrimers: Comparison of the release kinetics of covalently conjugated drug and non-covalent drug inclusion complex. Adv Drug Deliv Rev, 57:2203-14.

Patri AK, Majoros IJ, Baker JR. 2002. Dendritic polymer macromolecular carriers for drug delivery. Curr Opin Chem Biol, 6:466-71.

Quintana A, Raczka E, Piehler L, et al. 2002. Design and function of a dendrimer-based therapeutic nanodevice targeted to tumor cells through the folate receptor. Pharm Res, 19:1310-6.

Rudovsky J, Botta M, Hermann P, et al. 2006. PAMAM dendrimeric conjugates with a Gd-DOTA phosphinate derivative and their adducts with polyaminoacids: the interplay of global motion, internal rotation, and fast water exchange. Bioconjug Chem, 17:975-87.

Shellock FG, Kanal E. 1999. Safety of magnetic resonance imaging contrast agents. J Magn Reson Imaging, 10:477-84.

Swanson SD, Rosen MS, Agranoff BW, et al. 1997. Brain MRI with laserpolarized 129Xe. Magn Reson Med, 38:695-8.

Swanson SD, Rosen MS, Coulter KP, et al. 1999. Distribution and dynamics of laser-polarized (129)Xe magnetization in vivo. Magn Reson Med, 42:1137-45

Talanov VS, Regino CAS, Kobayashi H, et al. 2006. Dendrimer-based nanoprobe for dual modality magnetic resonance and fluorescence imaging. Nano Letters, 6:1459-63

Thomas TP, Majoros IJ, Kotlyar A, et al. 2005. Targeting and inhibition of cell growth by an engineered dendritic nanodevice. J Med Chem, 48:3729-35

van Buchem MA, Tofts PS. 2000. Magnetization transfer imaging. Neuroimaging Clin N Am, 10:771-88, ix.

Venditto VJ, Regino CA, Brechbiel MW. 2005. PAMAM dendrimer based macromolecules as improved contrast agents. Mol Pharm, 2:302-11.

Wiener EC, Brechbiel MW, Brothers H, et al. 1994. Dendrimer-based metal chelates: a new class of magnetic resonance imaging contrast agents. Magn Reson Med, 31:1-8. 